\title{
Emerging Personal Intelligence in Collective Goals: Data Analysis on the Bottom-Up Approach from PKM to OKM
}

\author{
Shahrinaz Ismail ${ }^{1}$, and Mohd Sharifuddin Ahmad ${ }^{2}$ \\ ${ }^{1}$ Universiti Kuala Lumpur (UNIKL), Malaysia,shahrinaz@miit.unikl.edu.my \\ ${ }^{2}$ Universiti Tenaga Nasional (UNITEN), Malaysia, sharif@uniten.edu.my
}

\begin{abstract}
There is a pattern of personal intelligence seen emerging from the concept of agent-mediated personal knowledge management (PKM) in achieving the organisational collective goals. This paper presents the results of analyses of related surveys implemented to prove this emergence, which include a quantitative analysis supported by a qualitative analysis on surveys across three main industries in Malaysia, namely manufacturing, service and education. From these analyses, we discovered that the emergence of personal intelligence is embedded within the collaborative interactions among software agents, and among agents and human knowledge workers. We show that our hypotheses discussed in this paper, which is supported by the results of the surveys manifest some organisational knowledge management (OKM) practice as a consequence of the agent-mediated PKM processes. Future work recommended to proving these hypotheses include the development of agent-based system that animates these theories in the real working environment.
\end{abstract}

Keywords: Personal knowledge management, personal intelligence, organisational knowledge management, software agent.

\section{INTRODUCTION}

With the shift of focus from organisational knowledge management (OKM) to personal knowledge management (PKM), recent studies emerge in looking from the angle of bottom-up approach instead of the traditional top-down approach of OKM. Studies do not stop in terms of social sciences alone, but also in terms of technical and agent intelligence. Taking this advantage, our current study focuses on agentmediated PKM processes, which results in the emergence of personal intelligence from the patterns among tasks delegated to software agents by human knowledge workers.

This paper discusses the emergence of personal intelligence, justified by the quantitative and qualitative data gathered in 2011 across three main industries, based on the following hypotheses:

H1: Agent-mediated PKM can be replicated.

H2: The replicated agent-mediated PKM represents a function of an individual knowledge worker's intelligence in an organization.

H3: The replicated agent-mediated PKMs overlap to reveal tasks for a common goal.

H4: The overlapping replicated agent-mediated PKMs can be integrated to manifest an agentmediated OKM.

\section{RELATED WORKS}

\section{A. PKM and OKM}

Myint (2004) and Zhang (2008) believe that individual knowledge workers are important to an organisation, hence the need to investigate the bottom-up approach of organisational knowledge management (OKM), with personal knowledge management (PKM) supporting the processes of the OKM. Despite the gap between PKM and OKM, researchers in the fields of internet technologies attempt to fill this gap by exploring the essential skills for PKM. Pettenati et al. (2007) and Razmerita et al. (2009) investigated the Web 2.0 technologies and tools used by knowledge workers in managing personal knowledge, and suggested that these tools should be used not only at individual level but also at organisational level.

The important aspect argued by researchers across the literature in this domain is the "people factor.' The PKM processes are defined in terms of networking, e.g. finding people who share the same social interest, sharing knowledge, collaborating, extending and extrapolating, and joining community of practice, since the core focus of PKM is 'personal inquiry', which is a quest to find, connect, learn and explore (Verma, 2009). Nonetheless, individual knowledge workers still perform different processes of PKM and often with different approaches at different times, depending on the situations. Yet, there are still similarities in the patterns of the processes, 
since the goals of performing the processes would eventually lead to the common collective goal. Even though PKM supports individual knowledge workers rather than establishing an organisational approach (Razmerita et al., 2009), the PKM environments integrate individual work environments and the facilitating infrastructure to support joint creation, distribution, sharing and application of knowledge (Martin, 2000). In other words, PKM can collectively contribute to OKM because knowledge is a source of competitive advantage at organisational level as well as at individual level (Razmerita et al., 2009).

\section{B. Agent-mediated PKM Processes}

On a more technical side, researchers are turning to software agents in mediating the processes of PKM because agents are claimed to be able to "carry out all the actions and exhibit all the behaviors within a knowledge flow" (Newman \& Conrad, 2000). As claimed by Ismail and Ahmad (2011) and supported by Apshvalka (2005), the four processes of PKM that can be mediated by software agents are: get/retrieve, understand/analyse, share, and connect. These processes are aligned with the PKM processes suggested by previous authors, such as Grundspenkis (2007), Martin (2000), Avery et al. (2001), Pettenati et al. (2007), and Razmerita et al. (2009), but with more focus on PKM over computer and internet technologies.

On top of that, the four processes of PKM depend on cognitive enablers, such as the method of performing tasks (i.e. method), how knowledge sources are identified (i.e. identify), how decision is made on the approach to take in seeking knowledge experts (i.e. decide), and the drive for knowledge workers to seek knowledge experts (i.e. drive) (Ismail \& Ahmad, 2011). These enablers are derived and analysed from previous researchers, such as Agnihotri and Troutt (2009) and Schwarz (2006).

\section{Personal Intelligence and Software Agents}

Being the domain under artificial intelligence, software agents are expected to be 'intelligent' with capabilities to re-act and pro-act on given situations. Among the capabilities and features of software agents expected in this study of personal intelligence are autonomy, reactive, proactive, able to communicate, adaptive, goal-oriented, capable to cooperate, reason, and flexible, as adapted from Paprzycki and Abraham (2003). In a separate case, personal intelligence is seen as one of the five layers that constitute collective intelligence, where the layer deals with enabling users (Solachidis et al., 2010). Similar to the software agent conceptual framework, Solachidis et al. (2010) personal intelligence exists within a restricted 'environment', such as event, user, content capture, terminal, and network.

Personal intelligence also involves the abilities to: recognize personally-relevant information from introspection and from observing oneself and others; form that information into accurate models of personality; guide one's choices by using personality information where relevant; and systematize one's goals, plans, and life stories for good outcomes (Mayer, 2008). In relating this concept with software agents, the intelligence of the agents would depend on the agents to be rational. Mayer (2008) also mentioned personal intelligence as having 'cumulative decisions' that helps in that person's well-being, which this study is investigating while focusing on the collective goals in achieving OKM.

\section{METHODOLOGY}

A questionnaire and an interview survey were conducted in 2011 to understand the pattern of PKM processes and how the PKM among knowledge workers could overlap in achieving the organisational goals. A total of 118 questionnaire surveys were answered and returned within 3 months, and 8 respondents were interviewed within 6 months. The respondents are from three main industries in Malaysia: Manufacturing, Service and Education.

The questionnaire design focused on gathering the information on how respondents manage their personal knowledge according to the four identified processes (i.e. get/retrieve knowledge, understand/ analyse knowledge, share knowledge, and connect to other knowledge or knowledge experts), and the enablers of the PKM processes that the respondents could verify (i.e. method, identify, decide, and drive). On the other hand, the interview consists of semi-structured questions that revolve around the same topics as the questionnaire survey, with additional questions that help validate the overlapping of tasks for a common goal, which would lead to the manifestation of OKM. The interview is also to justify and verify the findings gathered in the questionnaire survey.

From the results of these surveys, a framework is conceptualised to further understand how an agent-based system can be developed in mediating human PKM processes, and how personal intelligence can be animated from the agent-based framework to prove the proposed hypotheses. 


\section{RESULTS AND FINDINGS}

The responses received from the questionnaire survey show that knowledge workers generally perform the four processes of get/retrieve, understand/analyse, share and connect. Yet, the technology tools used for all these processes are not clearly identified except for the email. The email system is highly relied upon as the main tool for communication and knowledge sharing, since most organisations require knowledge workers to use office email for official tasks. Figure 1 shows the chosen options for each PKM process, highlighting the highest percentages of 'general search' for get/retrieve process, 'summarise' for understand/ analyse process, 'email' for share process, and 'email and online message' for connect process.

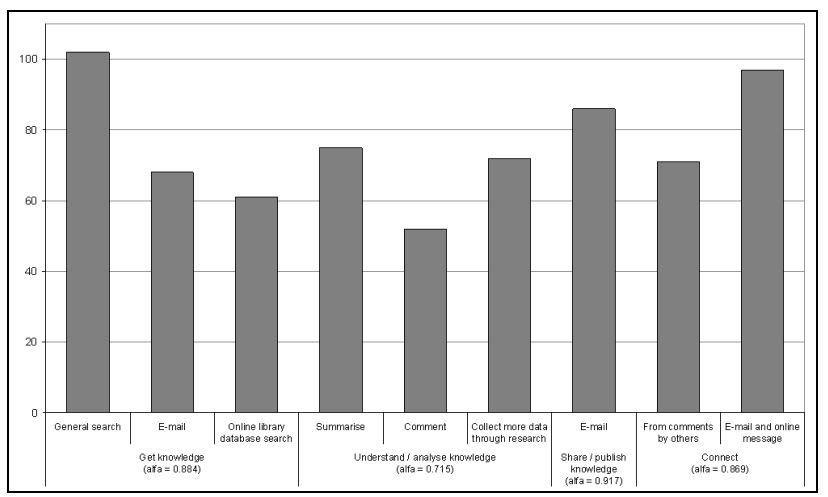

Figure 1. Knowledge Workers' PKM Processes $(n=118)$

In order to get or retrieve knowledge from the right source, knowledge workers need to identify and know who or where the sources are. The interview result provides some elaboration on this, which justifies the questionnaire response. Table 1 shows the top list of knowledge sources identified from the interview survey.

\begin{tabular}{|c|c|c|c|}
\hline Knowledge Sources & Education & Service & Manufacturing \\
\hline \multicolumn{4}{|c|}{ Other human knowledge workers } \\
\hline People & $\mathrm{Y}$ & $\mathrm{Y}$ & $\mathrm{Y}$ \\
\hline Professional (people) & $\mathrm{Y}$ & $\mathrm{Y}$ & Y \\
\hline Organisational people & $\mathrm{N}$ & Y & $\mathrm{N}$ \\
\hline
\end{tabular}

$\underline{\text { Knowledge repositories and databases }}$

\begin{tabular}{l|c|c|c} 
Personal database & N & Y & N \\
Organisational database & N & Y & Y \\
Organisational documents & N & Y & Y \\
\hline
\end{tabular}

Internet or World Wide/ Semantic Web

\begin{tabular}{|c|c|c|c|}
\hline Internet & $\mathrm{Y}$ & $\mathrm{Y}$ & $\mathrm{N}$ \\
\hline Online forums, blogs & $\mathrm{Y}$ & $\mathrm{N}$ & $\mathrm{N}$ \\
\hline $\begin{array}{l}\text { Journals, textbooks, } \\
\text { articles, magazines }\end{array}$ & $\mathrm{Y}$ & $\mathrm{N}$ & $\mathrm{N}$ \\
\hline
\end{tabular}

In terms of understanding or analysing knowledge, the results varies across industries, such as based on past experience or previous knowledge, information or recommendation by others, and reference by others that lead to a knowledge expert. Looking into the details of 'learning from past experience or previous knowledge', two interviewees mentioned different approaches: learn by observation and reviews from others (e.g. audience, people who listen to knowledge experts); and learn from documents (e.g. error logs and workflow documented in previous projects). However, the concept of referring to others' recommendations, whether 'they' are 'tacitly informative' or 'explicitly documented', still applies. These processes are part of the method and the way of identifying the right knowledge entity or source.

Looking at the situation across industries through these two surveys, it is discovered that PKM processes are applicable and practiced by common human knowledge workers. With this justification, a framework for a human knowledge worker and an agent-based environment in which the human works is drawn in a conceptualised nodal form, as shown in Figure 2. This diagram shows how the human knowledge worker delegates and interacts with software agents that execute the roles according to the PKM processes: GET, UNDERSTAND, and CONNECT. In order for the knowledge seeker to find the knowledge expert, the knowledge expert should be willing to share, hence the existence of an agent role called SHARE. 


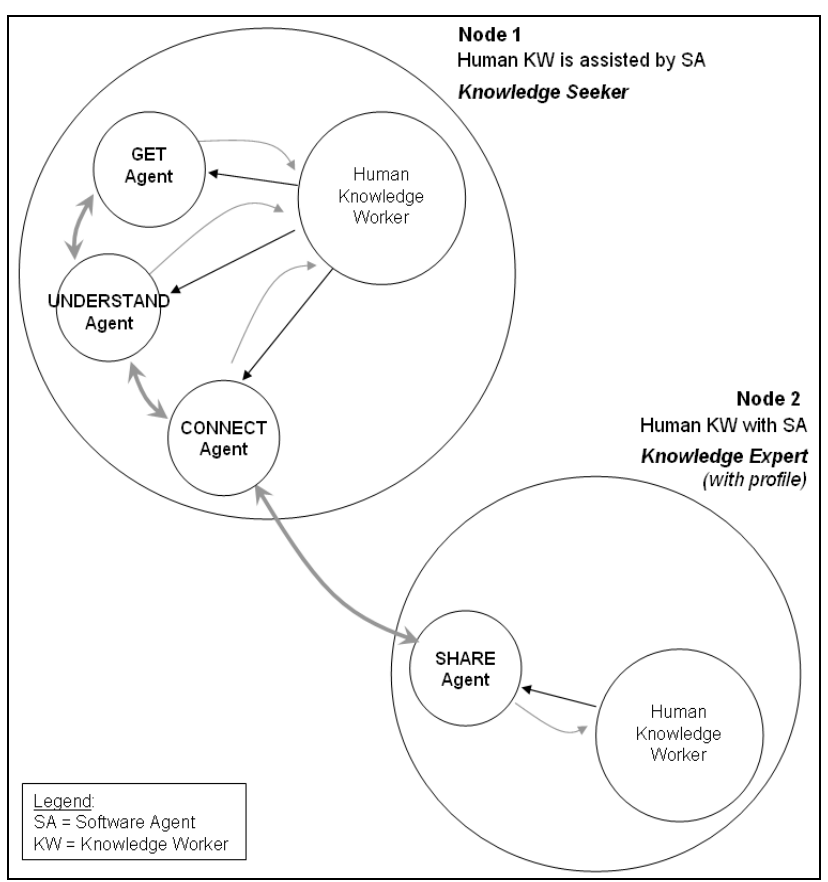

Figure 2. Overview of Agents' Roles in Mediating PKM Processes

Considering that the knowledge seeker may often be a person who shares knowledge with others, or in other words is also a knowledge expert to others, then all the agent roles of GET, UNDERSTAND, CONNECT and SHARE can be the mediating processes for the knowledge seeker. The interview survey results support this with respondents from middle management level admitting being the point of reference to others on certain subject matters and being the persons who seek others regarding subject matters which are not within their field of expertise. The significant findings include the capability to 'connect' to the one who has the expertise, provided that the expert is willing to 'share'.

Having said this, the single node of human knowledge worker shown in Figure 2 can be replicated into multiple nodes that would eventually form a network of connecting, and possibly socialising, nodes. Figure 3 shows this concept of 'social network' of nodes, with mediating PKM role agents from each node connecting to each other. In addition to this, the concept of personal intelligence can be seen emerging from the functions of the PKM role agents, with multiple nodes forming a group of human knowledge workers interacting within and across organisations.

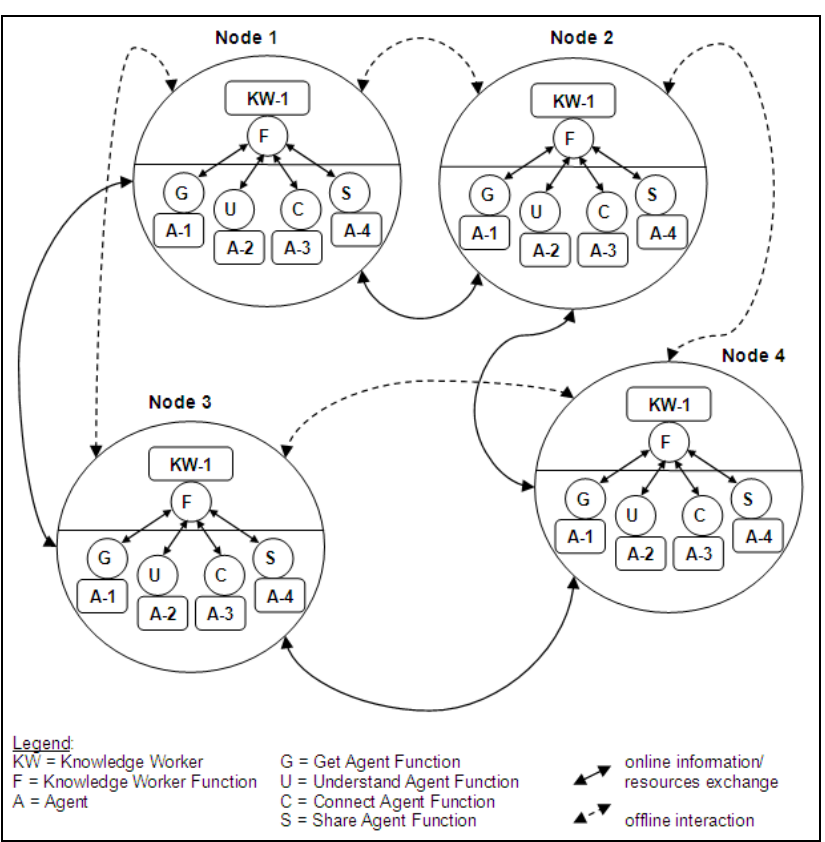

Figure 3. Multiple Nodes Replicated from a Single Multi-agent Environment for PKM Processes

With the support from the survey data analysis, Figure 2 and Figure 3 prove that "agent-mediated $P K M$ can be replicated" (H1) and "the replicated agent-mediated PKM represents a function of an individual knowledge worker's intelligence in an organisation" $(\mathrm{H} 2)$.

The interview survey reveals the overlapping of tasks and PKM patterns among knowledge workers in organisations. A respondent from manufacturing industry verified that it is a norm to have at least two knowledge workers involved in the same project or task, to ensure that one will cover the other if the latter is not available when certain information is needed regarding the project/task. These overlapping tasks are meant to achieve a common goal of the department, which will further ensure the achievement of the collective goal of the organisation. This is supported by other respondents from the service and education industries, who observed the same pattern of overlapping tasks for a common goal. The questionnaire survey results support this in terms of having higher agreement on "collaborate" task (38\%) compared to "delegate" task $(36 \%)$ in response to the question on how the respondents perform a task.

The responses received from the interview survey also lead to the verification of overlapping personal goals in fulfilling personal KPIs, which leads to collective goals measurable through departmental KPIs followed by the organisational goals and KPIs. This indicates that KPI is a way to perceive the overlapping of tasks within a department and the integrated KPI (whether 
within department or across departments) would lead to the manifestation of the organisational KPI.

If the intersection of common organisational tasks of knowledge workers:

$$
\mathrm{KW}_{1} \cap \mathrm{KW}_{2} \cap \mathrm{KW}_{3} \cap \ldots \cap \mathrm{KW}_{\mathrm{N}-1} \cap \mathrm{KW}_{\mathrm{N}} \square \square \square
$$

can be measured by their individual KPIs:

$$
\mathrm{KPI}_{1}+\mathrm{KPI}_{2}+\mathrm{KPI}_{3}+\ldots+\mathrm{KPI}_{\mathrm{N}-1}
$$

then the collective processes of achieving the organisational $\mathrm{KPI}, \mathrm{KPI}_{\mathrm{O}}$, can be construed as the OKM process.

$$
\begin{aligned}
& \mathrm{KPI}_{\mathrm{O}}=\mathrm{KPI}_{1}+\mathrm{KPI}_{2}+\ldots+\mathrm{KPI}_{\mathrm{N}-1} \\
& \mathrm{KPI}_{\mathrm{O}} \square \square \square \mathrm{KW}_{1} \cap \mathrm{KW}_{2} \cap \ldots \cap \mathrm{KW}_{\mathrm{N}-1} \cap \\
& \mathrm{KW}_{\mathrm{N}}
\end{aligned}
$$

In the context of knowledge management, the personal goal derived from the personal tasks is achievable through effective PKM processes, which manifests the organisational KM processes if the PKM processes overlaps the personal goals of other knowledge workers.

Based on these findings, it is proven that "the replicated agent-mediated PKMs overlaps to reveal tasks for a common goal" (H3) and "the overlapping replicated agent-mediated PKMs can be integrated to manifest an agent-mediated OKM' (H4).

\section{CONCLUSION AND FURTHER WORK}

Instinctively, knowledge workers perform their individual tasks to achieve their vocational goals, which are normally connected to the organizational goals. However, given the variations of work problems, each of them implements the processes of achieving his/her own goals in many different ways. Such differences, while appear to be disconnected, follows a common pattern of get/retrieve, understand/analyse, share, and connect processes.

We propose an agent-based framework to implement these PKM processes in which a human knowledge worker works in symbiosis with his/her agent in a node. Such nodal structure represents an emerging personal intelligence that implements the knowledge worker's PKM processes.

Our findings from the survey analyses also indicate that there are overlapping tasks which knowledge workers perform in achieving their own goals. Such overlap manifests the organizational knowledge management (OKM) processes.

In our future work, we shall implement these findings in an agent-based platform to simulate the emerging personal intelligence of humanagent coupling as well as the manifestation of an OKM as a consequence of many PKMs.

\section{REFERENCES}

Agnihotri, R., \& Troutt, M.D. (2009). The effective use of technology in personal knowledge management: A framework of skills, tools and user context. Online Information Review, 33(2), 329-342.

Apshvalka, D., \& Wendorff, P. (2005). A framework of personal knowledge management in the context of organisational knowledge management. Proceedings of the 6th European Conference on Knowledge Management (ECKM). 34-41.

Avery, S., Brooks, R., Brown, J., Dorsey, P., \& O’ Connor, M. (2001). Personal knowledge management: framework for integration and partnerships. Proceedings of Association of Small Computer Users in Education Conference, 29-39.

Grundspenkis, J. (2007). Agent based approach for organization and personal knowledge modelling: Knowledge management perspective. Journal of Intelligent Manufacturing, 18, 451-457.

Ismail, S., \& Ahmad, M. S. (2011). Emergence of Social Intelligence in Social Network: A Quantitative Analysis for Agent-mediated PKM Processes. Proceedings of the Fifth ICIMu 2011

Martin, J. (2000). Personal Knowledge Management. Managing Knowledge: Case Studies in Innovation. Edmonton: Spotted Cow Press.

Mayer, J. D. (2008). Personal Intelligence. Imagination, Cognition and Personality, 27, 209-232.

Myint, D. (2004). PKM: the starting blocks for KM. Inside Knowledge Magazine, 7.

Newman, B. D., \& Conrad, K. W. (2000). A Framework for Characterizing Knowledge Management Methods, Practices, and Technologies. Proceedings of the Third International Conference on Practical Aspects of Knowledge Management (PAKM2000).

Paprzycki, M., \& Abraham, A. (2003). Agent Systems Today: Methodological Considerations. Proceedings of the International Conference on Management of e-Commerce and e-Government, 1-7.

Pettenati, M.C., Cigognini, E., Mangione, J., \& Guerin, E. (2007). Using social software for personal knowledge management in formal online learning. Turkish Online Journal of Distance Education, 8, 52-65.

Schwarz, S. (2006). A context model for personal knowledge management. Modeling and Retrieval of Context, Lecture Notes in Computer Science, 3946, Springer: Berlin/Heidelberg, 18-33.

Solachidis, V., Mylonas, P., Geyer-Schulz, A., Hoser, H., Chapman, S., Ciravegna, F., et al. (2010). Collective Intelligence Generation from User Contributed Content. Advances in Data Analysis, Data Handling and Business Intelligence, Studies in Classification, Data Analysis, and Knowledge Organization. Springer-Verlag: Berlin, 765-774.

Razmerita, L., Kirchner, K., \& Sudzina, F. (2009). Personal knowledge management: The role of Web 2.0 tools for managing knowledge at individual and organisational levels. Online Information Review, 33, 1021-1039.

Verma, S. (2009). Personal Knowledge Management: A Tool to Expand Knowledge about Human Cognitive Capabilities. IACSIT International Journal of Engineering and Technology, 1( 5), 435-438

Zhang, Z. (2008). Personalising organisational knowledge and organisationalising personal knowledge. Online Information Review, 33, 237-256. 